\title{
LEVEL OF SURGICAL REAPPROACH IN REFERENCE WOMEN'S HEALTH UNIT
}

Catharine Coelho da Silva Santos¹, Mauro Orlando Meurer Oliveira', Felipe Andreotta Cavagna', Luiz Henrique Gebrim¹, Jorge Yoshinori Shida ${ }^{1}$

${ }^{1}$ Hospital Pérola Byington - São Paulo (SP), Brazil.

Globally, breast carcinoma is the most commonly diagnosed cancer and the main cause of death by cancer among women. Its treatment requires a multidisciplinary approach, involving radiotherapists, oncologists and surgeons. Despite the advancements in therapy, surgery still plays an essential role in the treatment. A postoperative complication rate is expected, which ranges according to the type and extension of the procedure. The quality of surgical approach is mirrored by the measurement of direct results, such as morbidity and mortality of the patient. There are some indicators of quality that are usually used to assess a service, such as rate of complications, time of hospitalization, patient's satisfaction and rate of reoperation. Objective: To assess the surgical reapproach rates in a reference women's health unit - Hospital Pérola Byington (CRSM), comparing them with the data observed in a literature review from major global institutions. Methods: Literature review of retrospective studies carried out in major institutions and data from CRSM. Results: The main complications that lead to surgical reapproach include bleeding, infection, seroma, problems in the injury, matters related to reconstruction and others. From the CRSM database, of a total of 8,806 analyzed patients, about $48.2 \%$ were submitted to conserving surgery (CS), and 51.8\%, to mastectomy (MASTE). The rate of reoperation in CRSM in the past 8 years is $1.82 \%$. By confronting these data with the data review of ACS-NSQIP, we identified that patients submitted to MASTE with immediate reconstruction (IR) were more prone to undergoing a reoperation due to complications $(6.8 \%)$ in comparison to patients who underwent MASTE without IR (2.8\%) or CS with or without pedicular rotation (2.4\% and $0.7 \%$, respectively); the general rate of reoperation was $4.2 \%$. Conclusion: Even though the rate is low, considering the high number of surgeries carried out in the service, it is necessary to maintain the complication rates to a minimum. The possible causes for the significantly reduced volume of reapproaches in CRSM should be considered: The imaging team is prepared, allowing a more accurate location of tumor beds, for example, the department of non-palpable lesions (NPL), which is responsible for mammotome clip and needling. This allows the signaling of a tumor bed, before and/or after being submitted to neoadjuvant chemotherapy (NEO CT); number of MASTE being higher to the number of CS, therefore, with fewer chances of residual tumor in the tumor bed. Usually, patients arrive to our unit at an advanced tumor clinical stage, therefore, with indication for MASTE; presence of pathologists in the surgical unit available at the time of the procedure, therefore, intraoperative freezing, which allows to increase margins in the same surgical period, preventing reapproaches; Multidisciplinary team, including NEO CT. Integrated care protocols can significantly reduce the rates of postoperative complications. 